\title{
Progress towards recalibration of spectrographs
}

\author{
Akhil Kallepalli*†, Umair Soori*, David B James*, Mark A Richardson* \\ * Centre for Electronic Warfare, Information and Cyber \\ Cranfield University, Defence Academy of the United Kingdom \\ Shrivenham, United Kingdom \\ †Corresponding author: Akhil Kallepalli, a.kallepalli@cranfield.ac.uk
}

\begin{abstract}
The spectral resolution of a spectrograph depends on the input slit width, the diffraction grating grooves and the number of imaging sensor/detector pixels. Due to the proprietary nature of spectrograph designs, recalibration by end-users can be challenging. Most calibration procedures currently published are applicable to in-house instruments or spectrographs with access to the internal specifications. Narrowing the input slit improves the resolution but also reduces the throughput of the imaging system. We attempted to recalibrate an Offner-based spectrograph by using a larger detector plane (an imaging system with a larger sensor), to vary the distance along the focal plane; and by utilising lens optics. Basic experiments were conducted by varying the distance from the exit window and inserting a lens to magnify the spectrograph output onto the larger detector plane. We concluded that the calibration could not be achieved using simple optics within the scope of our experiments. This article addresses a gap in literature that does not present the research community with the unsuccessful steps that are not applicable to similar problem statements. The alternative would be to rely on reflective optics, but this approach may reduce portability.
\end{abstract}

Index Terms-Offner-based Spectrographs, Optical Equipment, Spectral Domain, Focal Plane Array, Diffraction Gratings

\section{INTRODUCTION}

Advances in imaging systems and focal plane arrays have facilitated the transition of remote sensing from single-band, panchromatic (black and white) images to spectral data cubes. This differentiation of light in the spectral domain facilitates multispectral and hyperspectral imaging. The primary advantage of hyperspectral imaging lies in its ability to acquire spectral information in narrow, contiguous bands [1], [2], providing diagnostic data that is useful in diverse domains spanning from agriculture to medicine [3]; e.g. to show how plant health varies in an area [1] or to measure oxygen perfusion and saturation in the blood and/or tissues [4].

Most surfaces and materials exhibit characteristic emission, reflection, transmission and/or absorption behaviour when irradiated with light. The detection of these phenomena provides information about the target. The objective of spectral imaging is to capture the response at defined wavelengths, providing a spectral signature of the target. This requires an apparatus to separate incident light as a function of its wavelength. The imaging system that captures the dispersed spectrum defines the nature of the spectral information. Multispectral cameras cover a limited number of non-contiguous channels and produce broadband images, whereas hyperspectral imaging systems offer significantly finer sampling or narrow bands [5].

Essentially, to create a hyperspectral dataset (the hypercube), imaging systems must accommodate four dimensions of information: two spatial, one spectral and one time domain. The time domain is accommodated by scanning either in the spectral or spatial domain [5]. In spaceborne or airborne systems, the spatial domain scan is achieved by the motion of the platform itself [6]; this is accomplished using a scanning mirror instead of physically moving the platform in this study. The step duration of the scanning mirror determines the exposure and spatial resolution of the hypercube.

\section{RECALIBRATION}

Like most commercial equipment, spectrographs usually have proprietary designs. With no access to the internal specifications, every spectrograph is calibrated to achieve a specific resolution. The primary motivation for our experiments is to investigate whether a spectrograph can be recalibrated without internal modifications, allowing the resolution of a spectrograph operating in a given spectral range to be varied. The evolution of the spectrograph began with a prism-based design, which guaranteed a 50\% throughput [7]. However, the disadvantages of this design included the line profile of the output spectra [7] and the high absorption of light by the prism materials, leading to a comparatively low throughput [5], [7], [8]. Later spectrograph designs replaced the prism with a transmissive or reflective grating, the latter achieving up to $85 \%$ peak efficiency [5] with simultaneous acquisition of a linearly-dispersed spectrum without post-processing [9]. Using one such blazed and aberration-corrected convex grating [9], the current Headwall Photonics HyperSpec ${ }^{\odot}$ VNIR spectrograph [10] covers the spectral range 380-1000 nm.

In most dispersive spectrographs, a prism or diffraction grating splits the incoming light as a function of its wavelength. The splitting is due to the different angles of diffraction for individual wavelengths. This dispersion causes the incidence of light on the detector plane, from the focusing mirror. Additional optics are responsible for focusing the light on the grating and channelling the light onto the focal plane of the camera. The Offner spectrograph is widely used because it has a simple design (typically three components) and reflective surfaces [5], [7]. The HyperSpec ${ }^{\oplus}$ VNIR utilises focusing and collimating mirrors to direct the light from the entrance slit 
on to the convex, reflective diffraction grating, which divides the light. The diffracted output is directed on to the detector plane by a focusing mirror. The reflective optics achieve a high throughput.

Earlier calibration exercises against various references have been reported. Spectral calibration has been achieved by the direct viewing of various gas lamps [9]. For sensors operating in the infrared region $(8-12 \mu \mathrm{m})$, the deep sky on a cloudless day is used for calibration [11]. However, direct exposure to intense light can damage the sensor of the imaging camera. We therefore use a Spectralon in our experiments [12].

The resolution $(\delta \lambda)$ achieved by a spectrograph is directly proportional to the slit width $\left(W_{s}\right)$ and spectral range $(\Delta \lambda)$, and is inversely proportional to the number $(n)$ and width $\left(W_{p}\right)$ of the detector pixels [7], as shown in (1).

$$
\delta \lambda \propto \frac{\Delta \lambda \times W_{s}}{n \times W_{p}}
$$

In our experimental scenario, we did not modify the slit width or spectral range. Therefore, when using two imaging systems, (1) can be simplified to (2).

$$
\frac{\delta \lambda_{\text {imager } 1}}{\delta \lambda_{\text {imager } 2}}=\frac{n_{2} \times W_{p 2}}{n_{1} \times W_{p 1}}
$$

This relation estimates the spectral resolution an imaging system can achieve. In our experiments, a finer spectral resolution could be achieved because the larger Edge imaging system has more spectral pixels than the Pixelfly camera. Both cameras are introduced in the following section and note that all intensity measures are in arbitrary units (a.u.).

\section{EXPERIMENTAL CONFIGURATION}

In this investigation, we examined the spectral output of the PCO Pixelfly CCD [13], to which the spectrograph has been calibrated, to form the reference baseline. A mercury lamp emitting six wavelengths (shown as peaks) was used as the source. The light was reflected off the Spectralon into the HyperSpec ${ }^{\odot}$ VNIR spectrograph. The calibrated spectral output of the spectrograph (Fig. 1) was compared, under similar experimental conditions, with that of the PCO Edge VNIR camera [14] (Fig. 2). This showed a similar trend of (spectral) peaks, with a few additional peaks. The output of the spectrograph at the exit window when exposed to a full spectrum halogen lamp confirmed the additional orders of diffraction; this explains the high intensity peak at the beginning of the spectral curve (Fig. 2).

The Edge camera was fitted with an adjustable C-mount adapter, allowing the focal plane distance to be varied in 1-mm intervals. The Edge camera was placed at the same distance as the calibrated Pixelfly camera. This yielded a poorly-formed peak (Fig. 2), requiring further investigation into the focal plane distance for an imaging system with a larger sensor. The focal plane distance was thus investigated in intervals of 1 $\mathrm{mm}$. The full width at half maximum (FWHM) was calculated simultaneously and plotted in terms of number of bands at each position, to determine the optimal distance between the

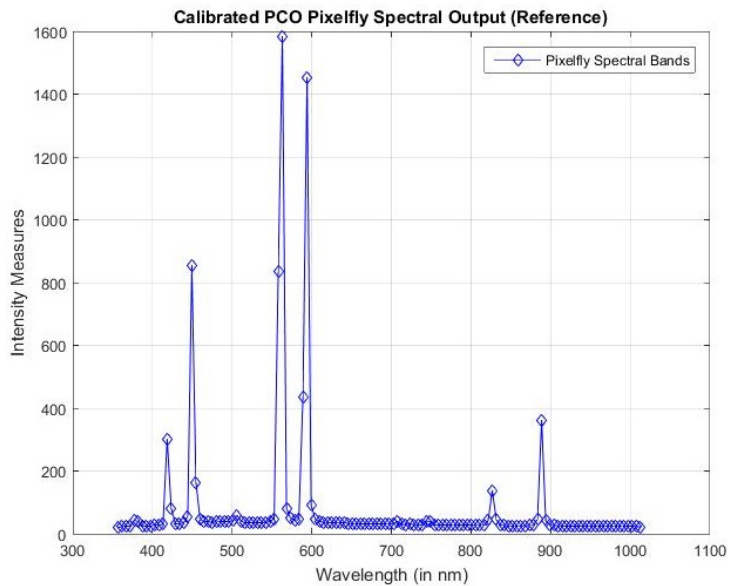

Fig. 1. The calibrated imaging system (PCO Pixelfly CCD sensor) shows six clearly defined peaks corresponding to the mercury lamp (source) reflected off the Spectralon.

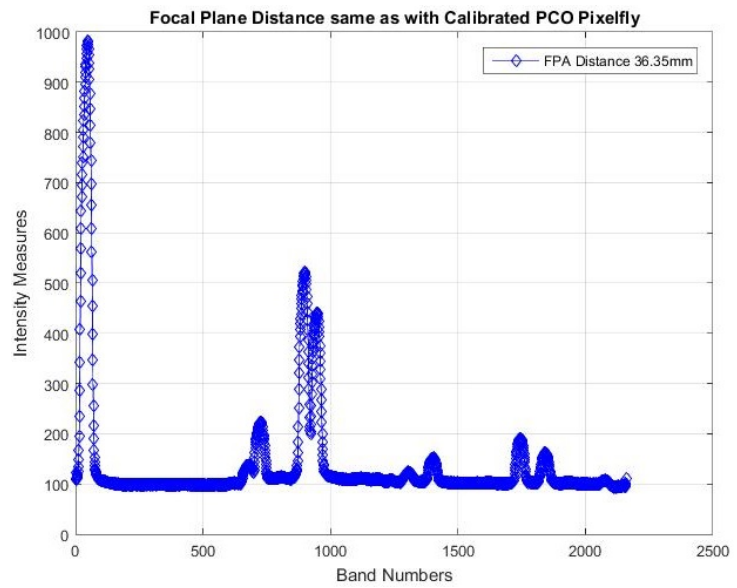

Fig. 2. The spectral response captured by the Edge sensor shows a similar pattern of the six peaks, and additional peaks that we consider to be the second order of diffraction.

camera and the exit window (Fig. 3). A single sharp peak is required to calculate the FWHM [11], [12], and we therefore selected the highest peak. The FWHM at each position was calculated (Fig. 3) and the optimal position was found at 43.35 $\mathrm{mm}$ from the exit window of the spectrograph $(7 \mathrm{~mm}$ from the optimal position of the Pixelfly) with the spectral curve shown in Fig. 4. At this optimal distance, the band numbers of the six peaks were plotted to determine the relationship between the cameras. Regression analysis was applied to the peak positions in order to calibrate the imaging systems. We found that the $546^{\text {th }}$ band of the Edge camera related to the first wavelength of the spectrograph range and the first band of the Pixelfly output.

With the distance established, the next step was to create a window/slit arrangement (Fig. 5) to prevent the high-intensity, undiffracted light and the additional orders of diffraction reaching the image plane (because the higher-resolution Edge sensor could be damaged by exposure to high-intensity light 


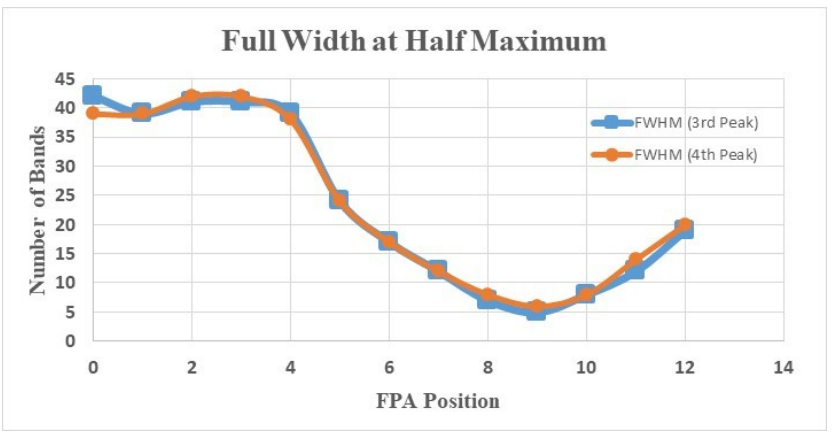

Fig. 3. Full width at half maximum comparison of the primary peak at 1$\mathrm{mm}$ intervals from the exit window. Position 9 is the image plane $43.35 \mathrm{~mm}$ from the spectrograph window. The peaks (third and fourth) are numbered excluding the high peak at the beginning of the spectral response.

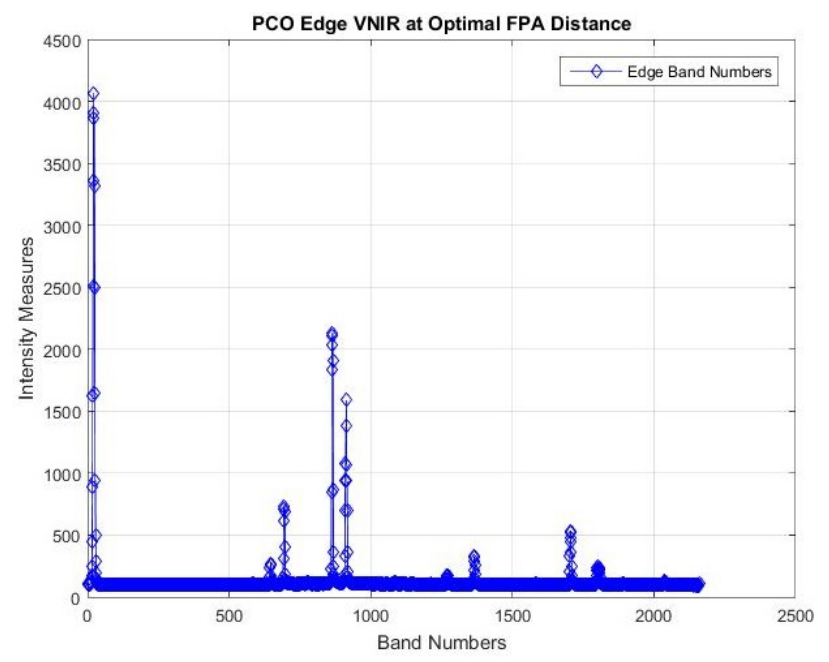

Fig. 4. At the optimal distance $(43.35 \mathrm{~mm})$, the spectral curve acquired by the PCO Edge CMOS imaging system show well defined peaks.

over time). A metal slit was centred in the path of the spectrograph output, allowing only the primary output (first order of diffraction) to reach the sensor (Fig. 6).

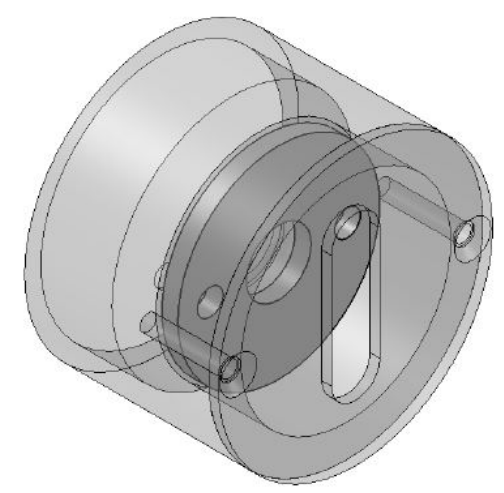

Fig. 5. Fabricated mount for the Edge adapter, including a blocking window and a slot for lens placement.

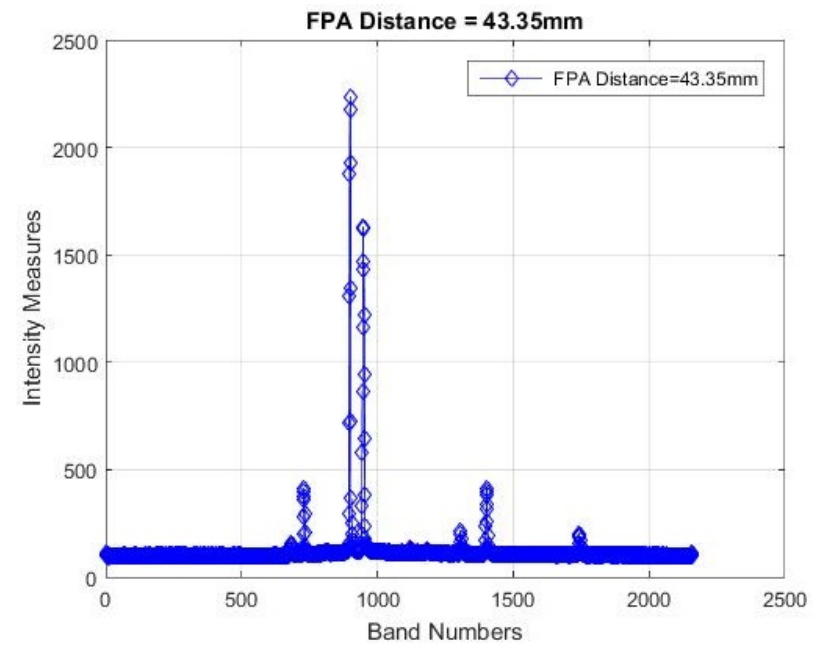

Fig. 6. Spectral information acquired by the PCO Edge sensor, with the blocking window in place.

\section{Current Status}

Having achieved the isolation of the primary order of diffracted light, a lens was placed in the holder assembly downstream from the exit window to block the additional peaks. The intention was to utilise the lens, placed at a predetermined distance, and project the magnified output onto the sensor plane. Comparing the sizes of the sensors on each camera, we hypothesised that a lens with the appropriate focal length could magnify the output of the spectrograph. The output of the spectrograph is calibrated to the dimensions of the Pixelfly sensor, which is smaller than the Edge CMOS sensor. Given that 2.4x magnification of the calibrated output was required, the $9 \mathrm{~mm}$ focal length lens (Edmund Optics DCX uncoated, $9 \mathrm{~mm}$ diameter $\times 9 \mathrm{~mm}$ focal length) was placed $12.5 \mathrm{~mm}$ from the spectrograph exit window. However, this simple adjustment did not yield any improvement in terms of spectral output. The resulting image could not be interpreted because the captured signal was effectively noise. The spectral information provided evidence of the failure of the lens to achieve the intended result.

In summary, our experiments involved the analysis of the spectral outputs of both imaging systems, followed by correlation of the calibrated and uncalibrated spectral outputs, the integration of a blocking window to remove additional orders of diffraction from the grating, and the use of a lens to magnify the output to the intended camera. The calibration of the focal plane distance is necessary during the recalibration of a spectrograph to a camera, whereas lens optics did not achieve the required outcome under our experimental conditions.

\section{DISCUSSION}

The investigation of focal plane distance can be considered an inevitable step in the recalibration of a spectrograph because the camera position needs to be confirmed before moving on to the next steps. We found that the use of an uncoated lens did not provide any benefits, and that placing 


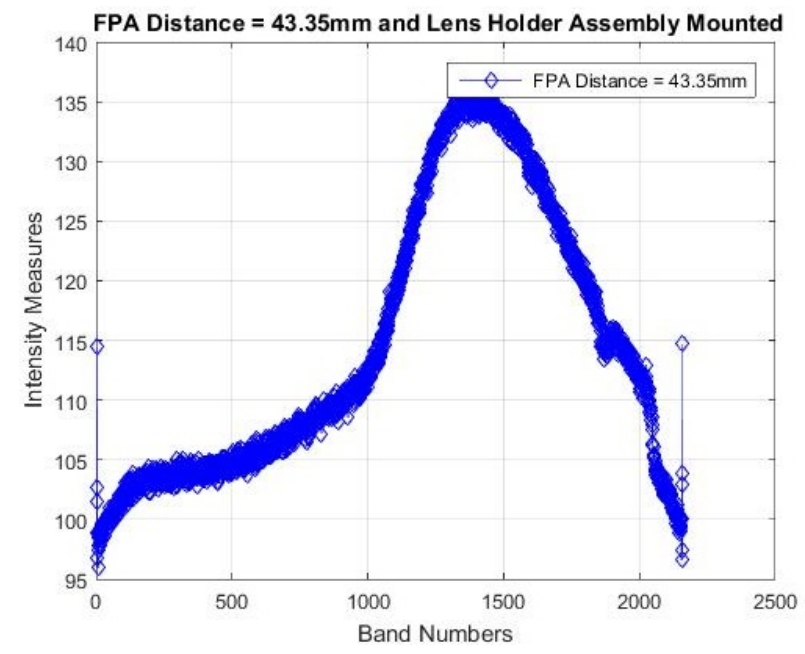

Fig. 7. At the known focal plane distance, the lens holder assembly including a blocking window and a $9 \mathrm{~mm}$ focal length lens.

a simple lens in the optical path of the spectrograph output did not facilitate recalibration. After adjusting the focal plane distance, the Edge CMOS sensor acquired an optimal spectral signal at $43.35 \mathrm{~mm}$, which is $7 \mathrm{~mm}$ further than the Pixelfly calibration distance, and a blocking window was used to remove the high-intensity, undiffracted light (Fig. 6) which has the potential to damage the sensor over time [7], [15]. Larger focal plane areas also encounter keystone and smile distortions when placed near the output window of the spectrograph [9].

The use of a simple lens distorted the spectral output of the spectrograph (Fig. 7). Knowledge of the distance and magnification requirements was not sufficient, in the scope of our experiments, to recalibrate the spectrograph externally. Varying the distances with the lens assembly in place did not yield any promising results either.

\section{CONCLUSiOn AND Future WORK}

This article outlines basic experiments carried out in an attempt to recalibrate a spectrograph using a small number of steps, but the anticipated results were not achieved. The highintensity peak we detected was caused by undiffracted white light from the grating (also referred to as ghost images/signals [7], [9], [15]), reaching the sensor. Order-sorting filters could be used to remove the additional orders of diffraction [12] thus avoiding the need for a physical blocking window.

We recommend a consideration of any of the above suggestions and/or an arrangement of mirrors for the recalibration of a spectrograph (specifically the Offner-design used in the COMPASS Hyperspectral Imager [12]). The loss of reflective optics on surfaces is low, and convex mirrors make the calibration steps easier. However, the use of additional mirrors at set distances will affect the portability of the spectrograph, and in most cases this is not feasible for laboratory experimentation. Ray tracing could be used to recreate these scenarios and build the results into the system design before hardware fabrication. As a final comment, the authors would like to share their belief that unsuccessful experimentation and negative results must be published for the benefit of the research community. Such literature supports the research philosophy and contributes to the growing body of supporting work for future applications.

\section{ACKNOWLEDGMENTS}

The authors would like to thank Dr Peter WT Yuen (Cranfield University) for his advice and access to equipment. We would also like to acknowledge Dr Kai-Ping Chuang (Industrial Technology Research Institute (ITRI)) and Dr Jane Hodgkinson (Cranfield University) for their advice during the experimental phase. Our colleagues, Mr Senthurran Selvagumar and Mr Murat Gunes, assisted our investigation of the problem statement and provided valuable input. We also acknowledge the input from Dr Richard Twyman for his assistance in the preparation of the manuscript.

\section{REFERENCES}

[1] G. A. Shaw and H.-h. K. Burke, "Spectral Imaging for Remote Sensing," Lincoln Laboratory Journal, vol. 14, no. 1, pp. 3-28, 2003.

[2] A. Kallepalli, A. Kumar, and K. Khoshelham, "Entropy based determination of optimal Principal Components of Airborne Prism EXperiment (APEX) Imaging spectrometer data for improved land cover classification," in ISPRS Technical Commission VIII Symposium. The International Archives of the Photogrammetry, Remote Sensing and Spatial Information Sciences, 2014, pp. 781-786.

[3] A. Kallepalli, A. Kumar, K. Khoshelham, and D. B. James, "Application of spectral and spatial indices for specific class identification in Airborne Prism EXperiment (APEX) imaging spectrometer data for improved land cover classification," in Proceedings in SPIE 10005, Earth Resources and Environmental Remote Sensing/GIS Applications VII, vol. 10005. SPIE, 2016, pp. 100 050Z-100 050Z-20.

[4] K. J. Zuzak, M. T. Gladwin, R. O. Cannon, and I. W. Levin, "Imaging hemoglobin oxygen saturation in sickle cell disease patients using noninvasive visible reflectance hyperspectral techniques: effects of nitric oxide." American journal of Physiology. Heart and Circulatory Physiology, vol. 285, pp. H1183-H1189, 2003.

[5] F. Vagni, "Survey of hyperspectral and multispectral imaging technologies," North Atlantic Treaty Organization, Research and Technology Organization, Neuilly-sur-Seine Cedex, France, Tech. Rep., 2007.

[6] T. M. Lillesand and R. W. Kiefer, "Concepts and Foundations of Remote Sensing," in Remote Sensing and Image Interpretation, 3rd ed. John Wiley \& Sons, Inc, 1994, ch. 1, pp. 1-44.

[7] J. James, Spectrograph Design Fundamentals, 1st ed. Cambridge: Cambridge University Press, 2007.

[8] S. Kaiser, B. Sang, J. Schubert, S. Hofer, and T. Stuffler, "Compact prism spectrometer of pushbroom type for hyperspectral imaging," in Proceedings of SPIE 7100, Optical Design and Engineering III. SPIE, 2008, pp. 710 014-710014-11.

[9] C. O. Davis, M. Kappus, J. Bowles, J. Fisher, J. Antoniades, and M. Carney, "Calibration, Characterization and first results with the Ocean PHILLS Hyperspectral Imager," in Proceedings of SPIE 3753, Conference on Imaging Spectrometry V, vol. 3753. Denver, Colorado: SPIE, 1999, pp. 160-167.

[10] "HyperSpec Imaging Series - User Manual," 2005.

[11] V. Farley, A. Vallieres, M. Chamberland, A. Villemaire, and J.-F. Legault, "Performance of the FIRST: a long-wave infrared hyperspectral imaging sensor," in Proceedings of SPIE 6398, Optically Based Biological and Chemical Detection for Defence III. SPIE, 2006, p. 11.

[12] J. Zadnik, D. Guerin, R. Moss, A. Orbeta, R. Dixon, C. G. Simi, S. Dunbar, and A. Hill, "Calibration procedures and measurements for the COMPASS hyperspectral imager," in Proceedings of SPIE 5425 Algorithms and Technologies for Multispectral, Hyperspectral, and Ultraspectral Imagery $X$, vol. 5425. SPIE, 2004, pp. 182-188.

[13] Pixelfly qe Manual, PCO Imaging, 2004.

[14] PCO.Edge Family Product Overview, 1st ed., PCO Imaging.

[15] J. R. Lakowicz, Ed., Instrumentation for Fluorescence Spectroscopy. Boston, MA: Springer US, 2006, pp. 27-61. 